\title{
Measuring Latent Causal Structure
}

\author{
Ricardo Silva \\ Department of Statistical Science \\ University College London \\ ricardo@stats.ucl.ac.uk
}

May 17, 2018

\section{Introduction}

Discovering latent representations of the observed world has become increasingly more relevant in the artificial intelligence literature Hinton and Salakhutdinov, 2006, Bengio and Cun, 2007. . Much of the effort concentrates on building latent variables which can be used in prediction problems, such as classification and regression.

A related goal of learning latent structure from data is that of identifying which hidden common causes generate the observations. This becomes relevant in applications that require predicting the effect of policies.

As an example, consider the problem of identifying the effects of the "industrialization level" of a country on its "democratization level" across two different time points. Democratization levels and industrialization levels are not directly observable: they are hidden common causes of observable indicators which can be recorded and analyzed. For instance, gross national product (GNP) is an indicator of industrialization level, while expert assessments of freedom of press can be used as indicators of democratization. Extended discussions on the distinction between indicators and the latent variables they measure can be found in the literature of structural equation models [Bollen, 1989] and errorin-variables regression [Carroll et al., 1995].

Causal networks can be used as a language to represent this information. We postulate a graphical encoding of causal relationships among random variables, where vertices in the graph representing random variables and directed edges $V_{i} \rightarrow V_{j}$ represent the notion that $V_{i}$ is a direct cause of $V_{j}$. Formal definitions of direct causation and causal networks are given by Spirtes et al. [2000] and Pearl 2000.

In our setup, we explicitly represent latent variables of interest as vertices in the graph. For example, in Figure 1 we have a network representation for the problem of causation between industrialization and democratization levels. This model makes assumptions about the connections among latent variables themselves: e.g., industrialization causes democratization, and the possibility of 


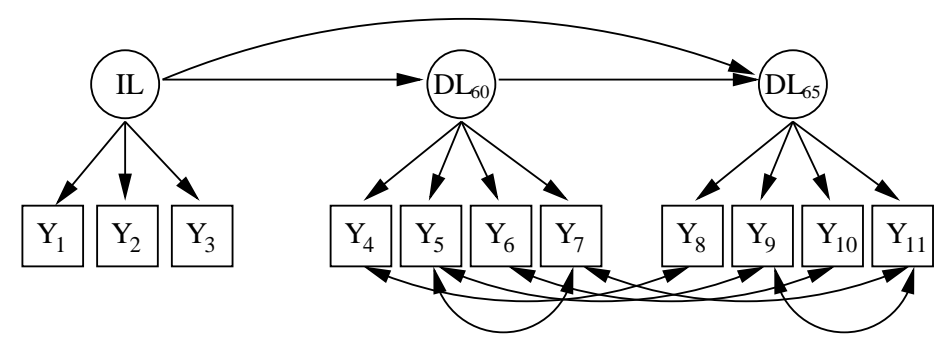

Figure 1: A causal diagram connecting industrialization levels $(I L)$ of a country, in 1960, to democratization levels in 1960 and $1965\left(D L_{60}\right.$ and $D L_{65}$, respectively). In our diagrams, we follow the standard structural equation modeling notation and use square vertices represent observable variables, circles represent latent variables (Bollen, 1989). The industrialization indicators are: $Y_{1}$, gross national product; $Y_{2}$, energy consumption per capita; $Y_{3}$, percentage of labor force in industry. The democratization indicators are: $Y_{4} / Y_{8}$, freedom of press; $Y_{5} / Y_{9}$, freedom of opposition; $Y_{6} / Y_{10}$, fairness of elections; $Y_{7} / Y_{11}$, elective nature of legislative body. Details are given by Bollen (1989).

unmeasured confounding between industrialization and democratization is not taken into account (which, of course, can be criticized and refined).

Following the mixed graph notation Richardson and Spirtes, 2002, Spirtes et al., 2000, Pearl, 2000], we also use bi-directed edges $V_{i} \leftrightarrow V_{j}$ to denote implicit paths due to latent common causes. That is, $V_{i} \leftrightarrow V_{j}$ denotes a set of causal paths (e.g., $V_{i} \leftarrow X \rightarrow V_{j}$ ) that originate from common causes that have been marginalized (such as $X$ in the previous example), as discussed in full detail by Richardson and Spirtes 2002. The distinction between "explicit" and "implicit" latent variables is problem dependent: if we do not wish to establish causal effects for some hidden variables, then they can be marginalized.

Establishing the causal connections among latent variables is an important causal question, but it is only meaningful if such hidden variables are connected to our observations. A complementary and perhaps even more fundamental problem is that of finding which latent variables are out there, and how they cause the observed measures.

This will be the main problem tackled in our contribution: given a dataset of indicators assumed to be generated by unknown and unmeasured common causes, we wish to discover which hidden common causes are those, and how they generate our data. Using a definition from the structural equation modeling literature, we say we are interested in learning the measurement model of our problem [Bollen, 1989].

In the context of the example of Figure 1] suppose we are given a dataset with 11 indicators, and wish to unveil the respective latent common causes and measurement model. Assuming Figure 1 as the unknown gold standard, we are successful if we predict that $\left\{Y_{1}, Y_{2}, Y_{3}\right\}$ are generated by a particular hidden 
common cause, $\left\{Y_{4}, \ldots, Y_{7}\right\}$ are generated by another hidden common cause and so on. Notice that interpreting the resulting latent variables and linking them to real entities and possible interventions requires knowledge of the domain. However, their existence and relationship to the observations follow from our data and assumptions, not from a posthoc interpretation.

The solution to this problem lies at the intersection of artificial intelligence techniques to infer causal structures, statistical models and the exploitation of assumptions commonly made in some applied sciences such as psychology and social sciences.

Success will depend on how structured the real-world causal network is and how valid our assumptions are. If the postulated true network that generated our data is not sparse, for instance, then there will be so many models compatible with the observed data that no useful conclusion can be made. This situation, however, is not different from the limitations of standard causal network discovery procedures (with no latent variables) Spirtes et al., 2000], which rely on the existence of many conditional independence constraints.

We describe our assumptions and a formal problem statement in full detail in Section 2. An algorithm to tackle the stated problem is provided in Section 3. Experiments are show in Section 4 followed by a Conclusion. Before that, however, we discuss what is the current common practice for unveiling the causal measurement structure of the world, and why they fall short on providing a reasonable solution.

\subsection{A motivating example}

Factor analysis is still the method of choice for suggesting hidden common cause models in the sciences. A detailed description of the method within the context of psychology and social sciences is given by Bartholomew and Knott 1999]. In this section, we will illustrate the weaknesses of factor analysis. This motivates the need for more advanced methods resulting from artificial intelligence techniques in causal discovery.

In a nutshell, the main assumption of factor analysis states that each observed variable $Y_{i}$ should be the effect of a set of latent factors $\mathbf{X} \equiv\left\{X_{1}, \ldots, X_{L}\right\}$ plus some independent error term $\epsilon_{i}$ :

$$
Y_{i}=\sum_{j=1}^{L} \lambda_{i j} X_{j}+\epsilon_{i}
$$

Variables are assumed to be jointly Gaussian, although this is not strictly necessary. The measurement model is given by the coefficients $\left\{\lambda_{i j}\right\}$ and variances of the error terms $\left\{\epsilon_{i}\right\}$. Learning the measurement model is the key task, which is required in order to understand what the hidden common causes should represent in the real-world. The factor analysis model is agnostic with respect to the causal structure of $\mathbf{X}$, but knowing the measurement model would also help us to learn the causal structure among latent variables [Spirtes et al., 2000]. In 
the following discussion, we will assume that we know how many latent variables exist, and then illustrate how such a widely used method is unreliable even under this highly favorable circumstance.

Given the observed covariance matrix of $\mathbf{Y} \equiv\left\{Y_{1}, \ldots, Y_{p}\right\}$, it is possible to infer the coefficients $\lambda_{i j}$ and the covariance matrix of $\mathbf{X}$, but not in an unique way. Without going into details, there are ways of choosing a solution among this equivalence class such that the measurement structure is as simple "as possible" (within the selection criterion of choice) Bartholomew and Knott, 1999]. Simplicity here means having many coefficients $\left\{\lambda_{i j}\right\}$ set to zero, indicating that each observed variable measures only a few of the latent variables. Getting the correct sparse structure is essential in order to interpret what the hidden common causes are. Notice that this corresponds to a directed causal network, where non-zero coefficients are encoded as directed edges in the graph.

Such methods will work when the true model that generated the data is in fact a "simple structure," or a "pure measurement model," in the sense that each observed variable has a single parent in the corresponding causal network. However, any deviance from this simple structure will strongly compromise the result.

We provide an example in Figure2, We generated data from a linear causal model that follows the causal diagram of Figure 2(a) 1 . Given data for the observed variables $Y_{1}, \ldots, Y_{6}$, we ideally would like to get a structure such as the one in 2(b), where the question marks emphasize that labels for the latent variables should be provided by background knowledge. Notice that in this contribution our aim is not to learn the structure connecting the latent variables, and the bi-directed edge in this case denotes an arbitrary causal connection.

Factor analysis fails to provide sensible answers. Figure2(c) shows a common outcome when we indicate that the model should have two hidden common causes. There exists no theory that provides a clear interpretation for these edges. Even worse, results can easily become meaningless. In Figure 2(d), we depict the result of exactly the same procedure, but where know we allow for three hidden common causes. The method we describe in our contribution is able to recover Figure 2(b).

\section{Problem statement and assumptions}

Assume that that our data follows a distribution $\mathcal{P}$ generated according to a directed acyclic causal graph (DAG) $\mathcal{G}$ Spirtes et al., 2000, Pearl, 2000] with observed nodes $\mathbf{Y}$ and hidden nodes $\mathbf{X}$. We also assume that the resulting distribution $\mathcal{P}$ is faithful to $\mathcal{G}$ Spirtes et al., 2000], that is, a conditional independence constraint holds in $\mathcal{P}$ if and only if it holds in $\mathcal{G}$ (using the common criterion of $d$-separation - please refer to Pearl (2000) for a definition and examples). These are all standard assumptions from the causal discovery literature.

Our more particular assumptions are

\footnotetext{
${ }^{1}$ Coefficients were generated uniformly at random on the inverval $[-1.5,-0.5] \cup[0.5,1.5]$ while variances of error terms were generated uniformly in $[0,0.5]$.
} 


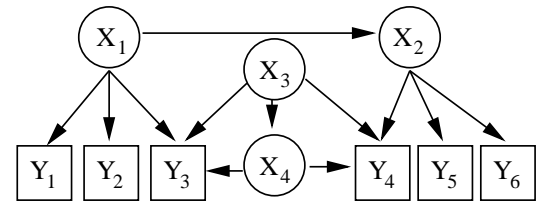

(a)

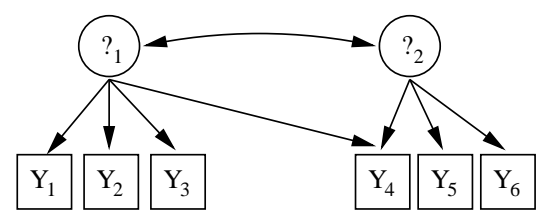

(c)

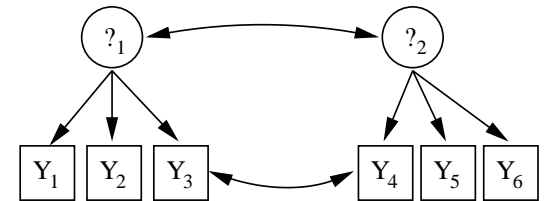

(b)

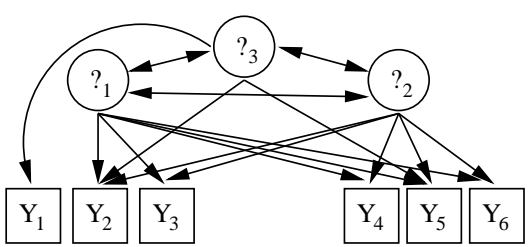

(d)

Figure 2: In (a), we show a synthetic structure from which we generated 200 data points. Our algorithm is able to reconstruct the causal graphical structure in (b), which captures several features of the original model. Bi-directed edges represent conditional association and the possibility of some unidentified set of hidden common causes of the corresponding vertices. Inferred latent variables are not labeled, but can then be interpreted from the resulting structure and the explicit assumptions discussed in Section 2 In (c), the resulting structure obtained with exploratory factor analysis using two latent variables and the promax rotation technique. In (d), the factor analysis result we got when attempting to fit three latent variables.

- no observed node $Y \in \mathbf{Y}$ is a parent in $\mathcal{G}$ of any hidden node $X \in \mathbf{X}$;

- each random variable in $\mathbf{Y} \cup \mathbf{X}$ is a linear combination of its parents, plus additive noise, as in Equation (10).

The first assumption is motivated by applications in structural equation modeling Bollen, 1989], where prior knowledge is used to distinguish between standard indicators and "causal indicators," which are causes of the latent variables of interest. Both of these assumptions can be relaxed to some extent, although any claims concerning the resulting causal structures learned from data will be weaker. Silva et al. [2006] discuss the details.

For the purposes of simplifying the presentation of this chapter, we also introduce the following two assumptions:

- no observed node $Y \in \mathbf{Y}$ is an ancestor in $\mathcal{G}$ of any other observed node;

- every pair of observed nodes in $\mathbf{Y}$ has a common latent ancestor 2 in $\mathcal{G}$;

These two assumptions can be dropped without any loss of generality [Silva et al., 2006], but they will be useful for presentation purposes. Notice that the latter

\footnotetext{
${ }^{2}$ As a reminder, this is not the same as having parents in common.
} 
assumption implies that there are no conditional independence constraints in the marginal distribution of $\mathbf{Y}$. As such, standard causal inference algorithm such as the PC algorithm [Spirtes et al., 2000] cannot provide any information.

Notice also that we do not assume any other form of background knowledge concerning the number of latent variables or particular information concerning which observed variables have common hidden parents.

Having clarified all assumptions on which our methods rely, the problem we want to solve can be formalized. Let the measurement model $\mathcal{M}$ of $\mathcal{G}$ be a graph given by all vertices of $\mathcal{G}$, and the edges of $\mathcal{G}$ that connect latent variables to observed variables. In order to be agnostic with respect to the causal structure among latent variables, we connect each pair of latent variables by a bi-directed edge as a general symmetric representation of dependency. Ideally, given the distribution $\mathcal{P}$ over the observed variables and that our assumptions hold, we would like to reconstruct $\mathcal{M}$. Since $\mathcal{P}$ has to be estimated from the data, it is of practical interest to use only features of $\mathcal{P}$ that can be easily estimated while also accounting for Gaussian distributions. As such, we rephrase our problem as learning $\mathcal{M}$ given $\Sigma$, the covariance matrix of $\mathbf{Y}$.

However, in general this is only possible if the true model entails that $\Sigma$ is constrained in ways that cannot be explained by other models. For instance, if there are more latent variables than observed variables, and each latent variable is a parent of all elements of $\mathbf{Y}$, then $\Sigma$ has no constraints and an infinite number of models will be compatible with the data.

Silva et al. 2006. formalize the problem by extracting only pure measurement submodels of the true model, subgraphs of $\mathcal{M}$ where each observed variable $Y$ has a single parent, and where this parent d-separates $Y$ from all other vertices of the submodel in $\mathcal{G}$. Such single-parent vertices are also called pure indicators. Moreover, the output of the procedure described by Silva et al. 2006] only generates submodels where each latent variable has at least three pure indicators. If such models exist, they can be discovered given $\Sigma$. The scientific motivation is that many datasets studied through structural model analysis and factor analysis support the existence of pure measurement submodels. As we mentioned in the previous section, methods for providing "simple structures" in factor analysis are hard to justify unless some pure measurement submodel exists. Therefore, it would be hard to justify factor analysis as a more flexible approach, since its output would be unreliable anyway. An important advantage of the causal discovery approach discussed here is that it knows its limitations.

Our contribution is to extend the work of Silva et al. by allowing several "impurities" in the output of our new procedure. To give an example where this is necessary, consider Figure 2(a) again. It is not possible to include both latent variables using the procedure of Silva et et al.: if latent variables $X_{1}$ and $X_{2}$, and their respective three indicators, are included, it turns out $Y_{3}$ is not d-separated from $Y_{4}$ by neither $X_{1}$ nor $X_{2}$. The best Silva et al. [2006] can do is to include, say, $X_{1}$ and its indicators, plus one of its descendants as an indirect indicator which does not violate the separations in the true model. For instance, the model with edges $X_{1} \rightarrow Y_{i}$, for $i \in\{1,2,3,5\}$ and no other variable, satisfies this condition. In contrast, the new procedure described here is able to generate 
Figure 2(b).

In practice, $\Sigma$ has to be estimated from data. In the discussion that follows, we assume that we know $\Sigma$ so that we can concentrate on the theory and the main ideas. Section 4 provides methods to deal with an estimate of $\Sigma$.

\subsection{Description of output}

Our output is a measurement pattern $\mathcal{M}_{P}$ which, under the above specified assumptions and given the population matrix $\Sigma$ of a set of observed variables $\mathbf{Y}$, provides provably correct causal claims concerning the true structure $\mathcal{M}$. The measurement pattern is a directed mixed graph with labeled edges (as explained below), with hidden nodes $\left\{L_{i}\right\}$ and observed nodes that form a subset of $\mathbf{Y}$. $\mathcal{M}_{P}$ includes directed edges from latent variables to observed variables, and bi-directed edges between observed variables.

Before introducing the new procedure in Section 3, we formalize the causal claims that a measurement pattern $\mathcal{M}_{P}$ provides:

1. each hidden variable $L_{i}$ in $\mathcal{M}_{P}$ corresponds to some hidden variable $X_{j}$ in $\mathcal{G}$. In the items below, we call this variable $X\left(L_{i}\right)$;

2. if $Y_{i}$ is not a child of latent variable $L_{j}$ in $\mathcal{M}_{P}$, then $Y_{i}$ is independent of $X\left(L_{j}\right)$ in $\mathcal{G}$ given its parents in $\mathcal{M}_{P}$;

3. given any pure measurement submodel of $\mathcal{M}_{P}$ with at least three indicators per latent variable, and a total of at least four observed variables, then at most one of the latent-to-indicator edges $L_{i} \rightarrow Y_{j}$ does not correspond to the true causal relationship in $\mathcal{G}$. That is, it is possible that for one pair, $X\left(L_{i}\right)$ is not a cause of $Y_{j}$ and/or the relationship is confounded;

The last item needs to be clarified with an example, since it is not intuitive. Let Figure 3(a) be a true causal structure from which we can measure the covariance matrix of $Y_{1}, \ldots, Y_{6}$. The structure reported by our procedure is the one in Figure 3(b). 5 out of 6 edges correspond to the correct causal statement, except $L_{2} \rightarrow Y_{6}$ (which should be confounded). We cannot know which one, but at least we know this is the case. As in any causal discovery algorithm Spirtes et al., 2000, Pearl, 2000, background knowledge is necessary to refine the information given by an equivalence class of graphical structures.

Finally, edges are labeled as "confirmed" (they do correspond to actual paths in the true graph) or "unconfirmed" (we cannot decide whether a corresponding path exists in the true graph). In the next section we clarify how "unconfirmed" edges appear. Unless otherwise stated, all other edges are "confirmed" edges.

\section{An algorithm for inferring an impure mea- surement model}

Let a one-factor submodel of $\mathcal{G}$ be a set composed of one hidden variable $X$ and four observed variables $\left\{Y_{A}, Y_{B}, Y_{C}, Y_{D}\right\}$ such that $X$ d-separates all four 


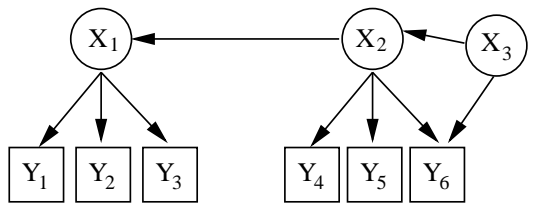

(a)

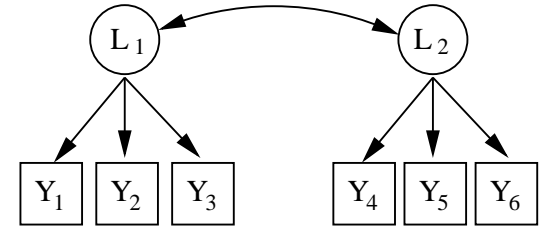

(b)

Figure 3: A covariance matrix obtained from the true model in (a) will result in the structure shown in (b). This structure should be interpreted as making claims about an equivalence class of models: in this case, at most one of the directed edges does not correspond to the exact claim that there is an unconfounded causal relationship between the latent variable and its respective child. But notice that there are only 7 models compatible with this claim, instead of the $2^{6}=32$ possibilities (each of the six relations $L_{i} \rightarrow Y_{j}$ being confounded or not) that the same adjacencies could provide.

observations in $\mathcal{G}$.

One-factor submodels play an important role in our procedure. A vertex $Y_{i}$ will be included in our output measurement pattern $\mathcal{M}_{P}$ if and only if it belongs to some one-factor submodel of $\mathcal{G}$. Also, $X$ will correspond to some output latent if and only if it belongs to some one-factor submodel. Figure 2(a) illustrates the concept: the sets $\left\{X_{1}, Y_{1}, Y_{2}, Y_{3}, Y_{5}\right\}$ and $\left\{X_{2}, Y_{4}, Y_{5}, Y_{6}, Y_{1}\right\}$ are one-factor submodels. No one-factor submodels exist for $X_{3}$ and $X_{4}$.

This fact should not be surprising. It is well-known in the structural equation modeling literature that the following model is testable:

$$
Y_{i}=\lambda_{i} X+\epsilon_{i}
$$

where $i \in\{1,2,3,4\}$, and $X$ and $\left\{\epsilon_{i}\right\}$ are mutually independent Gaussian variables of zero mean. This corresponds to a Gaussian causal network with corresponding edges $X \rightarrow Y_{i}$. Adding an extra edge, and hence a new parameter, would remove one degree of freedom and make the model undistinguishable from models with two latent variables [Silva et al., 2006].

One way to characterize which constraints are entailed by this model is by writing down the tetrad constraints of this structure. If $\sigma_{i j}$ is the covariance of $Y_{i}$ and $Y_{j}$, and $\sigma_{X}^{2}$ is the variance of $X$, then for the model (21) the following identify holds:

$$
\sigma_{12} \sigma_{34}=\lambda_{1} \lambda_{2} \sigma_{X}^{2} \times \lambda_{3} \lambda_{4} \sigma_{X}^{2}=\lambda_{1} \lambda_{3} \sigma_{X}^{2} \times \lambda_{2} \lambda_{4} \sigma_{X}^{2}=\sigma_{13} \sigma_{34}
$$

Similarly, $\sigma_{12} \sigma_{34}=\sigma_{14} \sigma_{23}$. For a set of four variables $\left\{Y_{A}, Y_{B}, Y_{C}, Y_{D}\right\}$, we represent the statement $\sigma_{A B} \sigma_{C D}=\sigma_{A C} \sigma_{B D}=\sigma_{A D} \sigma_{B C}$ by the predicate $\mathcal{T}(A B C D)$. Notice this is entailed by the graphical structure, since the relationship does not depend on the precise values of $\left\{\lambda_{i}\right\}$ or $\sigma_{X}^{2}$.

For the causal discovery goal, however, the relevant concept is the converse: given observable constraints that can be tested, which causal structures 
are compatible with them? Concerning one-factor submodels, the converse has been proved 3 by Silva et al. (2006):

Fact 1 If $\mathcal{T}(A B C D)$ is true, then there is a latent variable in $\mathcal{G}$ that $\mathrm{d}$ separates $\left\{Y_{A}, Y_{B}, Y_{C}, Y_{D}\right\}$.

For example, in Figure 3 (a), $X_{1}$ d-separates $\left\{Y_{1}, Y_{2}, Y_{3}, Y_{4}\right\}$, although it is not a cause of $Y_{4}$. A result such as Fact 1 is important for discovering latent variables, but it is of limited use unless there are ways of ruling out the possibility that some latent variables are causes of some indicators. It turns out that the $\mathcal{T}(\cdot)$ constraint can also be used for this purpose.

Consider Figure 3(a) again. If we pick all three indicators of one latent variables along with some indicator of the other latent variables, we have a onefactor model that passes the conditions of Fact 1. One possibility is that all six indicators are pure indicators of a single latent cause: after all, each pair $\left\{Y_{A}, Y_{B}\right\}$ is d-separated by some single latent variable. However, this does not tell us whether the latent variable that separates one group is the same as the one that separates another group. This is clear from Figure 3 (a): $X_{1}$ d-separates any pair in $\left\{Y_{1}, Y_{2}, Y_{3}\right\} \times\left\{Y_{4}, Y_{5}, Y_{6}\right\}$. However, it does not d-separate any pair in $\left\{Y_{4}, Y_{5}, Y_{6}\right\} \times\left\{Y_{4}, Y_{5}, Y_{6}\right\}$. We have to deduce this information without looking at the true graph, but only at the marginal covariance matrix of $\mathbf{Y}$.

One way of discarding connections from latents to indicators, and deducing that two unobserved variables are not the same, is given by the following result:

Fact 2 Consider the observed variables $\left\{Y_{A}, Y_{B}, Y_{C}, Y_{D}, Y_{E}, Y_{F}\right\}$. If both $\mathcal{T}(A B C D)$ and $T(A D E F)$ are true, but $\sigma_{A B} \sigma_{D E} \neq \sigma_{A D} \sigma_{B E}$, then $Y_{A}$ and $Y_{D}$ cannot have any common parent in $\mathcal{G}$.

A detailed proof is given by Silva et al. (2006). The intuitive explanation is that, if $Y_{A}$ and $Y_{D}$ did have a common parent (say, $X_{A D}$ ), then this latent variable would be precisely the one, and only one, responsible by both constraints $\mathcal{T}(A B C D)$ and $\mathcal{T}(A D E F)$. It would not be hard to show that this would imply $\sigma_{A B} \sigma_{D E}=\sigma_{A D} \sigma_{B E}$, contrary to the assumption.

Notice that these two results are already enough to find a pure measurement submodel. The general skeleton of the procedure is to find a partition $\left\{\mathbf{M}_{1}, \ldots, \mathbf{M}_{C}\right\}$ such that $\cup_{i=1}^{C} \mathbf{M}_{i} \subseteq \mathbf{Y}$ and

1. elements in $\mathbf{M}_{i}$ are d-separated by some hidden variable (using Fact 1);

2. elements in $\mathbf{M}_{i}$ and $\mathbf{M}_{j}$ cannot have common parents (using Fact 2).

Many more details need to be figured out in order to build an equivalence class of pure measurement models with three indicators per latent variable,

\footnotetext{
${ }^{3}$ To avoid unnecessary repetition, from now on we establish the convention that all results use the assumptions of Section 2 without explicitly mentioning them in the theoretical development.
} 
but this is the general idea. What is missing from this procedure is a way of coping with impure measurement models so that a structure such as the one in Figure 2(b) can be obtained. We now introduce the first theoretical results that accomplish that.

\subsection{Finding impure indicators}

Consider what can happen if we observe the covariance matrix generated by the model of Figure 2(a). We know that there is no single latent variable that d-separates (say) $\left\{Y_{1}, Y_{2}, Y_{3}, Y_{4}\right\}$. However, we know that there is some hidden $L$ that d-separates $\left\{Y_{1}, Y_{2}, Y_{3}, Y_{5}\right\}$, as well as some hidden $L^{\prime}$ that d-separates $\left\{Y_{1}, Y_{4}, Y_{5}, Y_{6}\right\}$. So far, it could be the result of a graph such as the following:

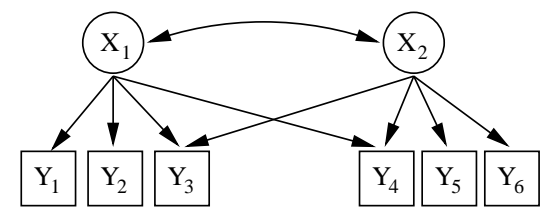

However, we cannot stop here and report this as a possible solution: we will get an inconsistent estimate for the covariance of the latent variables, which can lead to wrong conclusions about the causal structure of the latents. We would like to account for the possibility that the impurities arise not from our identified latents, but from some other source. This is the result summarized by Lemma 3:

Lemma 3 Consider the observed variables $\left\{Y_{A}, Y_{B}, Y_{C}, Y_{D}, Y_{E}, Y_{F}\right\}$. If the folllowing predicates are true:

$$
\mathcal{T}(A B C E), \mathcal{T}(A B C F), \mathcal{T}(A D E F), \mathcal{T}(B D E F)
$$

and the following predicates are false

$$
\mathcal{T}(A B E F), \mathcal{T}(A B C D), \mathcal{T}(C D E F)
$$

then in the corresponding causal graph $\mathcal{G}$, we have that:

- $\mathcal{G}$ contains at least two different latent variables, $L_{1}$ and $L_{2}$;

- $L_{1}$ d-separates all pairs in $\left\{Y_{A}, Y_{B}, Y_{C}\right\} \times\left\{Y_{D}, Y_{E}, Y_{F}, L_{2}\right\}$, except $Y_{C} \times$ $Y_{D}$;

- $L_{2} d$-separates all pairs in $\left\{Y_{A}, Y_{B}, Y_{C}, L_{1}\right\} \times\left\{Y_{D}, Y_{E}, Y_{F}\right\}$, except $Y_{C} \times$ $Y_{D}$;

- $Y_{C}$ and $Y_{D}$ have extra hidden common causes not in $\left\{L_{1}, L_{2}\right\}$. 


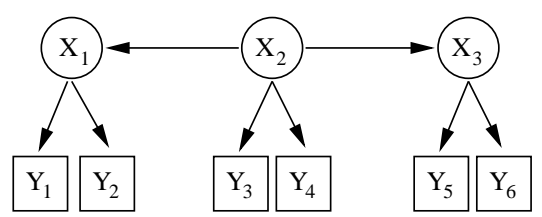

(a)

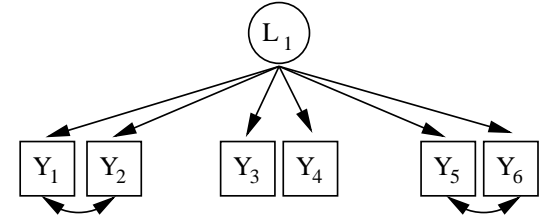

(b)

Figure 4: A model such as the one in (a) generates the measurement pattern in (b). Notice that the indication of extra hidden common causes, as represented by, e.g., $Y_{1} \leftrightarrow Y_{2}$, does not imply that these unrepresented causes are independent of the represented ones. Notice also that if the edge $X_{1} \leftarrow X_{2}$ was switched in (a) to $X_{1} \rightarrow X_{2}$, the corresponding pattern would still be the one in (b). In this case, it is clear that both edges $L_{1} \rightarrow Y_{1}$ and $L_{1} \rightarrow Y_{2}$ are not representing the actual causal directions of the true graph. However, the corresponding measurement pattern claim is about pure submodels. In this case, a pure submodel could be the one containing $Y_{1}, Y_{3}, Y_{4}$ and $Y_{5}$ only. One edge, $L_{1} \rightarrow Y_{1}$, still does not explicitly indicate the confounding given by $X_{1}$, but this is compatible with the measurement pattern description.

A formal proof of a slightly more general result is given by Silva 2006]. The core argument is as follows. The existence of $L_{1}$ and $L_{2}$ follows from Fact 1 and the constraints $\mathcal{T}(A B C E)$ and $\mathcal{T}(A D E F)$. That $L_{1} \neq L_{2}$ follows from Fact 2 and the fact that $\mathcal{T}(A B E F)$ is false. The other d-separations follow from Fact 1 and the given tetrad constraints. Finally, if $Y_{C}$ and $Y_{D}$ did not have any other hidden common cause, we could not have both $\mathcal{T}(A B C D)$ and $\mathcal{T}(C D E F)$ falsified at the same time, contrary to our hypothesis.

Notice that we never claim that the implicit latent variables represented by bi-directed edges are independent of the discovered latent variables. Figure 4 illustrates a case.

The second type of impurity we will account for nodes that have more than one represented latent parent.

Lemma 4 Consider the observed variables $\left\{Y_{A}, Y_{B}, Y_{C}, Y_{D}, Y_{E}, Y_{F}, Y_{G}\right\}$. If the folllowing predicates are true:

$$
\mathcal{T}(A B C K), \text { for } K \in\{D, E, F, G\} ; \mathcal{T}(K E F G), \text { for } K \in\{A, B, C, D\} ;
$$

and the following predicates are false

$$
\begin{aligned}
\mathcal{T}\left(K_{1} K_{2} K_{3} K_{4}\right), \text { for }\left\{K_{1}, K_{2}\right\} \subset\{A, B, C\},\left\{K_{3}, K_{4}\right\} \subset\{E, F, G\} ; \\
\mathcal{T}(A D E F), \mathcal{T}(A B D E)
\end{aligned}
$$

then in the corresponding causal graph $\mathcal{G}$, we have that:

- $\mathcal{G}$ contains at least two different latent variables, $L_{1}$ and $L_{2}$;

- $L_{1} d$-separates all pairs in $\left\{Y_{A}, Y_{B}, Y_{C}, Y_{D}\right\}$; 


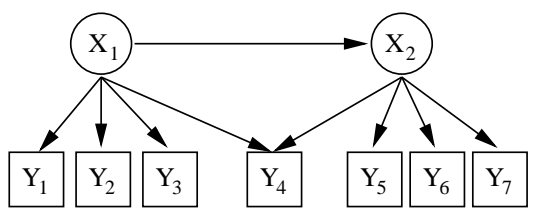

(a)

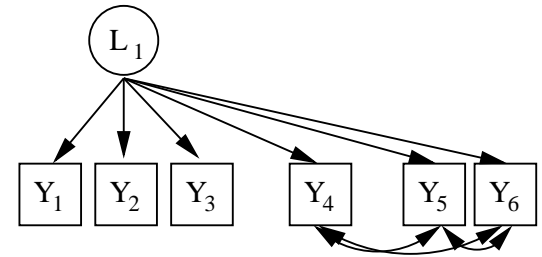

(b)

Figure 5: The graph in (a) can be rebuild exactly. However, without a fourth indicator of $X_{2}$ (e.g., $Y_{7}$ ), this latent variable can not be detected and the result would be the graph in (b).

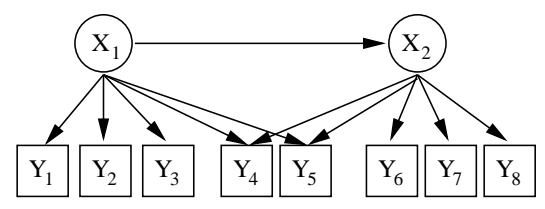

(a)

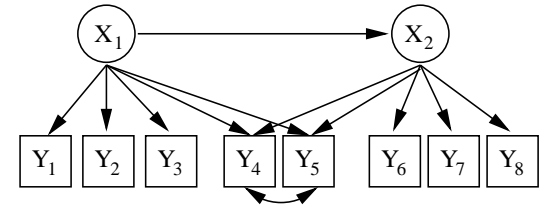

(b)

Figure 6: The graph in (a) is not fully identifiable with tetrad constraints only. A conservative measurement pattern needs to report the structure in (b).

- $L_{2} d$-separates all pairs in $\left\{Y_{D}, Y_{E}, Y_{F}, Y_{G}\right\}$;

- $L_{1} d$-separates all pairs in $\left\{Y_{A}, Y_{B}, Y_{C}\right\} \times\left\{Y_{E}, Y_{F}, Y_{G}, L_{2}\right\}$, but not $Y_{D} \times$ $L_{2}$;

- $L_{2} d$-separates all pairs in $\left\{Y_{A}, Y_{B}, Y_{C}, L_{1}\right\} \times\left\{Y_{E}, Y_{F}, Y_{G}\right\}$, but not $Y_{D} \times$ $L_{1}$;

The nature of this result complements the previous one: instead of searching for evidence to remove edges from latents into indicators, this result provides identification of edges that cannot be removed.

The argument again exploits Facts 1 and 2. A more detailed proof is given by Silva 2006. Notice the need for extra indicators in this case: this is another illustration of the need for one-factor models for each latent variable. Without $Y_{7}$ in the example of Figure 5 (a), the result would be the measurement pattern of Figure 5 (b).

Notice that if there are indicators that share more than one common parent in $\mathcal{G}$, we cannot separate them (i.e., avoid a bi-directed edge) even if their parents are identified in the model using tetrad constraints only. Figure 6 illustrates what the measurement pattern should report. Using higher-order constraints than tetrad constraints might be of help in this situation Sullivant and Talaska, 2008], but this is out of the scope of the current contribution.

To summarize:

- Fact 1 provides the evidence to include latent variables; 
- Fact 2 provides the evidence to distinguish between different latent variables;

- Lemma 3 allows the removal of extra edges from latents into indicators and proves the necessity of some bi-directed edges;

- Lemma 4 proves the necessity of some edges from latents into indicators, but does not prove the necessity of adding some bi-directed edges;

\subsection{Putting the pieces together}

So far, we have described how to identify particular pieces of information about the underlying causal graph. While those results allow us to identify isolated latent variables and to remove or confirm particular connections, we need to combine such pieces within a measurement pattern. Unlike the procedure of Silva et al. [2006], this pattern should be able to represent several pure measurement submodels within a single graphical object and to possibly include more latent variables than any pure model.

In this section, we assume that we have the population covariance matrix $\Sigma$. We start by finding groups of variables that are potential indicators of a single latent variable. We first build an auxiliary undirected graph $\mathcal{H}$ as follows:

INITIALPASS: this procedure returns an undirected graph $\mathcal{H}$.

1. let $\mathcal{H}$ be a fully connected undirected graph with nodes $\mathbf{Y}$;

2. for all groups of six variables $\left\{Y_{A}, Y_{B}, Y_{C}, Y_{D}, Y_{E}, Y_{F}\right\}$ that form a clique in $\mathcal{H}$, if $\mathcal{T}(A B C D)$ and $\mathcal{T}(A D E F)$ are true but $\sigma_{A B} \sigma_{D E} \neq \sigma_{A D} \sigma_{B E}$, remove the edge $Y_{A}-Y_{D}$;

3. if for a given $Y_{A}$ in $\mathcal{H}$ there is no triplet $\left\{Y_{B}, Y_{C}, Y_{D}\right\}$ such that $\mathcal{T}(A B C D)$ holds, then remove $Y_{A}$ from $\mathcal{H}$, since there will be no one-factor model including $Y_{A}$;

4. return $\mathcal{H}$.

Notice that if two vertices are not adjacent in $\mathcal{H}$, they cannot possibly be children of the same latent variable (it follows from Fact 2). This motivates us to look for one-factor models within cliques of $\mathcal{H}$ only. In Step 3, we discard variables not in one-factor models, since nothing informative can be claimed about them using our methods.

In the next step, we obtain a set of tentative subgraphs, where each subgraph contains a single latent variable and its indicators:

SingLeLATENTS: given $\mathcal{H}$, this procedure returns a set $\mathcal{S}$ of graphs with a single latent variable each.

1. initialize $\mathcal{S}$ as the empty set 
2. for each clique $\mathcal{C}$ in $\mathcal{H}$

3. $\quad$ if there is no $\left\{Y_{A}, Y_{B}, Y_{C}\right\} \subset \mathcal{C}$ and $Y_{D} \in \mathbf{Y}$ such that $\mathcal{T}(A B C D)$ holds, continue to next clique

4. $\quad$ create a graph $\mathcal{G}_{i}$ with latent vertex $L_{i}, i=|\mathcal{S}|+1$, and with children given by $\mathcal{C}$

5. for each $\left\{Y_{A}, Y_{B}\right\} \subset \mathcal{C}$, if there is no $Y_{C} \in \mathcal{C}$ and $Y_{D} \in \mathbf{Y}$ such that $\mathcal{T}(A B C D)$ is true, then add edge $Y_{A} \leftrightarrow Y_{B}$ to $\mathcal{G}_{i}$. Mark this edge as "unconfirmed";

6. $\quad$ add the new graph to $\mathcal{S}$;

7. return $\mathcal{S}$.

By Fact 1, every single latent variable created in this procedure exists in $\mathcal{G}$. The rationale for Step 5 is that $L_{i}$ does not d-separate $Y_{A}$ and $Y_{B}$. It is possible to confirm many of such edges using an argument similar to Lemma 4 , but we leave out a detailed analysis to simplify the presentation 4 .

Finally, all single graphs are unified into a coherent measurement pattern:

FindMeasurementPattern: returns a measurement pattern $\mathcal{M}_{P}$ given $\mathcal{S}$.

1. let $\mathcal{M}_{P}$ be the union of all graphs in $\mathcal{S}$, where all latents are connected by bi-directed edges

2. for every pair $\left\{\mathcal{S}_{i}, \mathcal{S}_{j}\right\} \subset \mathcal{S}$ do

3. $\quad$ consider all triplets $\left\{Y_{A}, Y_{B}, Y_{C}\right\} \subset \mathcal{S}_{i} \cup \mathcal{S}_{j}$ such that $\mathcal{T}(A B C D)$ holds for some $Y_{D}$. If such triplets are also in $\mathcal{S}_{i} \cap \mathcal{S}_{j}$, set the children of $L_{j}$ firmed"

to be children of $L_{i}$ and discard $L_{j}$. Set all $L_{i} \rightarrow Y_{k}$ to be "unconif $Y_{k}$ is not in $\mathcal{S}_{i} \cap \mathcal{S}_{j}$. Continue to next pair;

4. for every pair $\left\{Y_{C}, Y_{D}\right\} \subset \mathcal{S}_{i} \cap S_{j}$, add "unconfirmed" edge $\mathcal{Y}_{C} \leftrightarrow Y_{D}$ to $\mathcal{M}_{P}$. If Lemma 3 can be applied to $\left\{Y_{C}, Y_{D}\right\}$ where $\left\{Y_{A}, Y_{B}\right\} \subset S_{i}$ and $\left\{Y_{E}, Y_{F}\right\} \subset S_{j}$, then remove edges $L_{j} \rightarrow Y_{C}$ and $L_{i} \rightarrow Y_{D}$ and $\operatorname{mark} \mathcal{Y}_{C} \leftrightarrow Y_{D}$ as "confirmed";

\footnotetext{
${ }^{4}$ An example: in Figure 5(b), all bi-directed edges can be confirmed, because each of $\left\{Y_{4}, Y_{5}, Y_{6}\right\}$ are separated from $\left\{Y_{1}, Y_{2}, Y_{3}\right\}$ by $L_{1}$. We can therefore isolate the failure of having a one-factor model composed of $\left\{L_{1}, Y_{1}, Y_{2}, Y_{4}, Y_{5}\right\}$ down to the $Y_{4} \leftrightarrow Y_{5}$ edge.
} 


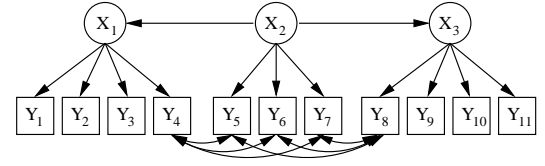

(a)

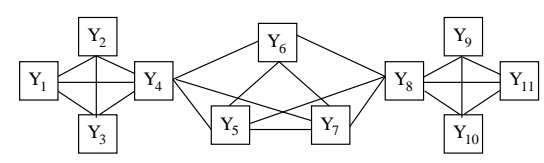

(b)

Figure 7: With the true graph being (a), we obtain two cliques of variables $\left\{Y_{4}, Y_{5}, Y_{6}, Y_{7}\right\}$ and $\left\{Y_{5}, Y_{6}, Y_{7}, Y_{8}\right\}$ in $\mathcal{H}$ (Figure (b)), since we can discover that $Y_{4}$ and $Y_{8}$ are indicators of different variables. However, these two cliques are related to the same true latent $X_{2}$ and have to be merged. The side-effect is that we cannot confirm the edges $L_{2} \rightarrow Y_{4}$ and $L_{2} \rightarrow Y_{8}$, although we know both cannot possibly exist at the same time.

5. if $Y_{j}$ has more than one parent, mark all directed edges $L_{i} \rightarrow Y_{j}$ unsupported by Lemma 4 as "unconfirmed";

6. return $M_{P}$.

The justification for most steps follows directly from our previous results. To understand Step 3, however, we need an example. In Figure 7(a), we have a true model. We can separate $Y_{4}$ from $Y_{8}$ using Fact 2. The result of FirstPass is the graph $\mathcal{H}$ shown in Figure $7(\mathrm{~b})$. Sets $\left\{Y_{4}, Y_{5}, Y_{6}, Y_{7}\right\}$ and $\left\{Y_{5}, Y_{6}, Y_{7}, Y_{8}\right\}$ are cliques in $\mathcal{H}$, but they refer to the same latent variable $X_{2}$. There will be edges $L_{2} \rightarrow Y_{4}$ and $L_{2} \rightarrow Y_{8}$ in the measurement pattern, but they will not be confirmed edges. Notice that there might be ways of removing $L_{2} \rightarrow Y_{4}$ and $L_{2} \rightarrow Y_{8}$, but they are out of the scope of our paper. Our goal is not to provide complete identification methods, but to show the main tools and the difficulties of learning impure measurement models.

\section{Experiments}

In this section, we illustrate how the theory can be applied by analyzing two simple datasets.

In practice, we will not know $\Sigma$, but only an estimate obtained from a sample. Robust statistical procedures to score models and test constraints from finite samples are described at length by Silva et al. [2006].

In the following experiments, we assume data are multivariate Gaussian. Wishart's tetrad test can be used to evaluate $\mathcal{T}(\cdot)$, which we accept as true if the p-value for the test is greater or equal to 0.05 Silva et al., 2006]. In the SingleLatents procedure, for each clique $\mathcal{C}$ we add extra bi-directed edges to $\mathcal{G}_{i}$ by a greedy search procedure: we look at each pair of variables and evaluate the Bayesian information criterion (BIC, Schwarz [1978]) for the model with the added edge. If the best model is better than the current one, we keep the edge. Otherwise, we stop modifying $\mathcal{G}_{i}$. An analogous procedure is performed to add bi-directed edges in FindMeAsurementPattern. 


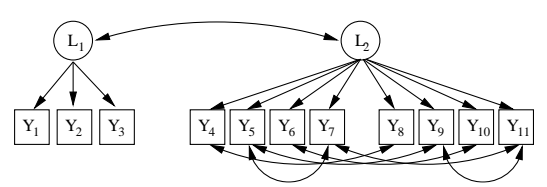

(a)

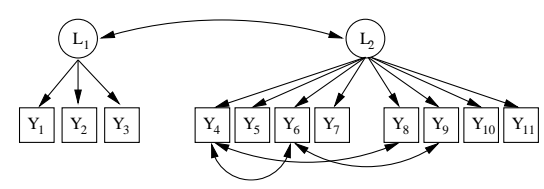

(b)

Figure 8: In (a), the measurement pattern that corresponds to the gold standard. In (b), the result of our procedure. All directed edges are correct. With small sample sizes, the BIC score tends to produce models simpler than expected, so it is not surprising that the model lacks several of the bi-directed edges.

In the worst-case scenario, the procedure scales at an exponential in the number of variables due to the necessity of finding cliques in a graph (the SiNGLELATENTS procedure). The examples are small and sparse enough so that this is not a problem. Some heuristics for larger problems are described by Silva et al. [2006].

\subsection{Democratization and industrialization example}

This is the study described at the beginning of Section 1 and discussed by Bollen [1989]. A sample of 75 countries was collected. We will discuss the outcome of our procedure and how it relates to the gold standard of Figure 1

If the true model is indeed Figure 1 and if we had access to an oracle that could answer exactly which tetrad constraints hold and do not hold in the true model, then the result of our algorithm would be Figure 8(a). The result obtained with our implementation is shown in Figure 8(b). With only 75 samples, it is not surprising that the BIC score tends to produce models with fewer edges than expected. Still, the model reveals a lot of information present in the expected pattern. It also suggests ways of extending the procedure, such as allowing for the background knowledge that some variables have the same definition, but recorded over time. Recall that the resulting model was obtained without any extra information.

\subsection{Depression example}

The next dataset is a depression study with five indicators of self-steem $(S E L F)$, four indicators of depression (DEPRES) and three indicators of impulsiveness $(I M P U L S)$. This dataset is one of the examples that accompany the LISREL software for structural equation modeling. The depression data and the meaning of the corresponding variables can also be found at

- http://www.ssicentral.com/lisrel/example1-2.html

A theoretical gold standard is show in Figure 9(a). It is worth mentioning that, treated as a Gaussian model, this graphical structure does not fit the data: 


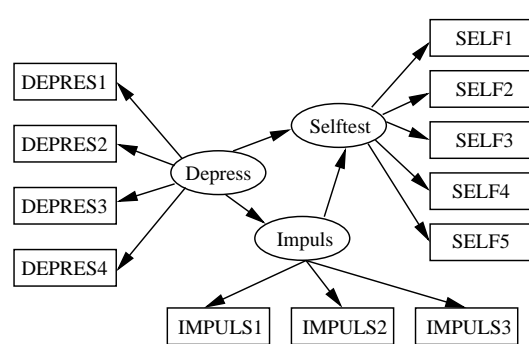

(a)

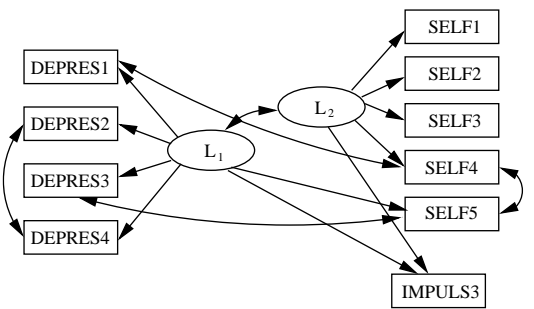

(b)

Figure 9: In (a), the gold standard of the depression study. The measurement pattern is precisely the same (except for the latent connections). In (b), the result of our procedure. The inferred model cannot contain the impulsiveness latent variables, as it turns out the correlation of IMPULS1 and IMPULS2 with other variables are statistically too close to zero at a 0.10 level.

the chi-square score is 122.8 with 51 degrees of freedom. The sample size is 204 .

Our result is shown in Figure 9(b). It was impossible to find a hidden common cause for the indicators of impulsiveness: the correlations of IMPULS1 and IMPULS2 with the other items were just too low, and those items had to be discarded. The only major difference against the gold standard was assigning SELF5 with the incorrect latent parent (the role of IMPULS3 in the solution is compatible with the properties of a measurement pattern). Given the number of bi-directed connections into SELF5, however, this indicator seems particularly problematic.

It is relevant to stress that in this study, the indicators are ordinal (in a 0 to 4 scale), not continuous. We were still able to provide relevant information despite using a Gaussian model. In future work, methods to deal with ordinal data will be developed. The theory for ordinal data is essentially identical, as discussed by Silva et al. 2006]. However, non-Gaussian models need to be used, which increases the computational cost of the procedure considerably.

\section{Conclusion}

Learning measurement models is an important causal inference task in many applied sciences. Exploratory factor analysis is a popular tool to accomplish this task, but it can be unreliable and causal assumptions are often left unclear. Better approaches are needed. Loehlin [2004] argues that while there are several approaches to automatically learn causal structure, none can be seem as competitors of exploratory factor analysis. Procedures such as the one introduced by Silva et al. [2006] and extended here are important steps that fill this gap.

The inclusion of impure indicators is an important step to make such approaches more generally applicable. As hinted in our discussion, other identification results to confirm or remove edges can be further developed. Higher- 
order constraints in the covariance matrix, besides tetrad constraints, are yet to be exploited Sullivant and Talaska, 2008]. Exploring the higher-order moments of the observed distribution (i.e., not only the covariance matrix) has been a successful approach to identify the causal structure of linear models Shimizu et al., 2006], but how to adapt them to discover a measurement model is still unclear. Finally, some progress on allowing for non-linearities has been made [Silva and Scheines, 2005], but more robust statistical procedures and further identification results are necessary.

\section{References}

D. Bartholomew and M. Knott. Latent Variable Models and Factor Analysis. Arnold Publishers, 1999.

Y. Bengio and Y. Le Cun. Scaling learning algorithms towards AI. Large Scale Kernel Machines, 2007.

K. Bollen. Structural Equations with Latent Variables. John Wiley \& Sons, 1989.

R. Carroll, D. Ruppert, and L. Stefanski. Measurement Error in Nonlinear Models. Chapman \& Hall, 1995.

G. Hinton and R. Salakhutdinov. Reducing the dimensionality of data with neural networks. Science, 313(5786):504-507, 2006.

J. Loehlin. Latent Variable Models: An Introduction to Factor, Path and Structural Equation Analysis. Lawrence Erlbaum, 2004.

J. Pearl. Causality: Models, Reasoning and Inference. Cambridge University Press, 2000.

T. Richardson and P. Spirtes. Ancestral graph Markov models. Annals of Statistics, 30:962-1030, 2002.

G. Schwarz. Estimating the dimension of a model. Annals of Statistics, pages 461-464, 1978.

S. Shimizu, P. Hoyer, A. Hyvärinen, and Antti Kerminen. A linear non-gaussian acyclic model for causal discovery. Journal of Machine Learning Research, 7: 2003-2030, 2006.

R. Silva. Principled selection of impure measures for consistent learning of linear latent variable models. NIPS Workshop on Causality and Feature Selection, 2006.

R. Silva and R. Scheines. New d-separation identification results for learning continuous latent variable models. Proceedings of the 22nd Interational Conference in Machine Learning, 2005. 
R. Silva, R. Scheines, C. Glymour, and P. Spirtes. Learning the structure of linear latent variable models. Journal of Machine Learning Research, 7:191246, 2006.

P. Spirtes, C. Glymour, and R. Scheines. Causation, Prediction and Search. Cambridge University Press, 2000.

S. Sullivant and K. Talaska. Trek separation for Gaussian graphical models. arxiv::0812.1938, 2008. 